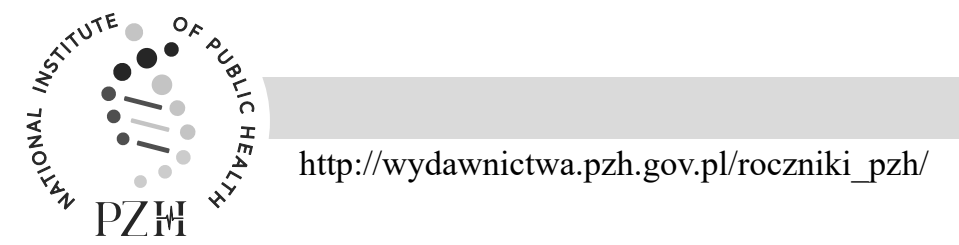

\title{
STUDY ON HEALTH RISK FACTORS AS A BASIS TO IMPLEMENT PROGRAMS THAT PROMOTE A NUTRITIONAL CULTURE IN STUDENTS OF THE LA SABANA UNIVERSITY
}

\author{
Sandra Rodriguez ${ }^{l}$, Mariagracia Martinez ${ }^{l}$, Franco Segnini $^{l}$, Valentina Cajiao ${ }^{l}$, \\ Iván Aivasovskyl, Luis Gustavo Celis ${ }^{l}$, Jaime Ayalal, Catalina Rozol, Amparo Russi ${ }^{2}$, \\ Vilma Giratá ${ }^{3}$, Carmen Cecilia Almonacid ${ }^{3}$ \\ ${ }^{1}$ School of Medicine - La Sabana University, Chía, Cundinamarca, Colombia \\ ${ }^{2}$ Centro Médico Country, Bogota, Colombia \\ ${ }^{3}$ Bacteriology Program - Colegio Mayor de Cundinamarca University, Bogota, Colombia
}

\begin{abstract}
Background. Nowadays the importance of lifestyles in the prevention of type 2 diabetes and the metabolic syndrome has been largely accertained.

Objective. The purpose of our work is to implement programs that promote a nutritional culture in adolescents and young adults of the La Sabana University.

Methods. The methodology entailed, after the corresponding informed consent, taking measures of the triceps and supraescapular skinfolds, waist circumference, body mass index (BMI), lean mass, and fat mass. Fasting blood samples were also taken to quantify cholesterol, triglycerides, high density lipoprotein (HDL) and low density lipoprotein (LDL). Results. The results obtained show that of the 165 students, $10.3 \%$ were underweight, $13.93 \%$ were overweight and $0.6 \%$ were obese. With regards to gender, $4.8 \%$ of the men and $9 \%$ of the women were overweight, $3 \%$ of the men and $7.2 \%$ of the women were underweight, and $0.6 \%$ of the women were obese. The blood chemistry showed that $30 \%$ had hypercholesterolemia, 18\% hypertriglyceridemia, 17\% reported low HDL levels and 67\% reported high LDL levels. Of all the cases studied, $40 \%$ are at risk of a metabolic syndrome. $60 \%$ claimed not to practice any physical activity - especially women who reported $44.70 \%$.

Conclusions. These findings have allowed us at the institution to implement a culture of healthy habits. The have also allowed us to identify students with risk factors for type 2 diabetes and metabolic syndrome. This is why the cardiometabolic monitoring and control based on healthy eating and physical activity are important.
\end{abstract}

Key words: health promotion, disease prevention, obesity, non-communicable diseases, healthy lifestyle

\section{INTRODUCTION}

Nowadays, the importance has been recognized of the lifestyles on the health level of the adolescent population, which over the years has become an important target group for the study of the different factors that are most relevant to the development of non-transmissible chronic diseases - some of the factors being the diet, physical activity and lipid profile [10, 25]. Bearing in mind that the eating behavior is one of the aspects having a major impact on the health and physical composition of the population, several studies have shown the importance and high prevalence of the effects of inadequate eating habits in the population, such as the frequent consumption of carbohydrates, saturated fats, low consumption of fruits, vegetables and the lack of implementation of eating timetables $[9,23]$.

According to the estimation and projection of the National Administrative Department of Statistics (DANE), by 2020 the Colombian young adult population aged $16-21$ years will be $5,113,289$. This is a representative and relevant number, given that, a ccording to the Pan-American Health Organization, adolescents and young adults represent a key factor for the social, economic and political progress of all countries [11, 15, 21].

In general, and according to various studies, all age groups are affected by inadequate eating behaviors. However, in adolescents and young adults, 
these unhealthy behaviors make them more sensitive because they are at the peak of their growth and have larger energy requirement, in addition to a low physical activity and a high level of sedentary lifestyles [7, 14]. That is why the implementation of different programs on healthy lifestyles in educational institutions is one of the most effective methods in the prevention of noncommunicable diseases (NCDs) such as obesity, type 2 diabetes mellitus and metabolic syndrome - these being the most significant causes of death worldwide, as they represent, as a whole, $70 \%$ of annual deaths [8].Therefore, adolescence must be seen as an age of opportunity and not only as one of vulnerability, and it is time to influence the acquisition of adequate habits and healthy lifestyles $[12,13]$.

Obesity and the metabolic syndrome are complex and heterogeneous clinical entities with a strong genetic component, the expression of which is influenced by environmental, social, cultural and economic factors, among others. The parallel increase in the frequency of obesity and the metabolic syndrome is a worldwide phenomenon and Colombia is no exception. In addition to this, these pathologies are important risk factors for the development of type 2 diabetes, coronary artery disease and cerebrovascular disease due to arteriosclerosis, which are the main causes of death in our country $[12,13]$.

The control of these metabolic alterations directly affects the morbidity and mortality of many diseases. Notwithstanding, at present there are no effective prevention, diagnosis and treatment strategies for most of the cases. For these reasons, obesity and the metabolic syndrome have become a serious public health problem in Westernized countries [12, 13, 22].

Public health plays a fundamental role, both in the identification of determining factors and their possible solutions as well as in the implementation of demographic measures for their control and the evaluation of their effectiveness. However, given the relevance of these conditions on the health of the community, it is necessary to find scientific strategies to shorten the times in the generation of knowledge and allow the design of prevention and treatment models. The goal will have been achieved as soon as these models are operable through assistance programs and the frequency of these entities is reduced [13, 22].

The objective of our work is to determine body composition, main habits and lifestyles in the study population, to the purpose of implementing nutritional, pedagogical and physical activity intervention programs to foster a nutritional culture in adolescents and young adults of the La Sabana University.

\section{MATERIAL AND METHODS}

This was a descriptive, cross sectional study devided in two phases with a representative randomly selected sample of the population of La Sabana University in Bogota, Colombia, consisting of 165 young students from different careers - most of them from the medicine school, aged $16-21$, who at the time of the analyzes were enrolled in the institution. The study also included volunteers with no history of drug addiction, alcoholism, mental illness, and no pacemaker users.

Before the survey, project participants signed the informed consents and the adequate review by the Ethics and Research Committee (project EICEA-68-2013). First phase of both quantitative and qualitative data collection and documenting was set up with the support of La Sabana University's research department [20]. In turn, the body composition was measured and taking the triceps and supraescapular skinfold using an adipometer, waist circumference, BMI, lean mass, fat mass, $\%$ fat, among others, with the Tanita Electronics Model TBF 310A impedance tester (Tanita Corporation). Blood samples were also taken with a previous fast of 12 hours, to determine the total cholesterol, triglycerides, HDL and LDL, using the Mindray BS-200 auto-analyzer (Annar Diagnostica Import SAS). Cardiovascular risk surveying was also conducted based on certified international formats, where data on the eating habits, exercise and major risk factors such as smoking and familial risk factors were collected.

Based on the results, and by relating them with the available evidence the second phase of the project were conducted, in which we analyzed the information gathered to finally formulate a series of recommendations to promote healthy habits and to reinforce ongoing measures established within the university's boudries.

\section{RESULTS}

The results obtained indicate that of a final sample of 165 students, (114 women and 51 men, aged 16-21 years), $75.1 \%$ presented a normal weight, $10.3 \%$ were underweight, $13.93 \%$ were overweight and $0.6 \%$ were obese, according to the standardized BMI data of the World Health Organization (WHO) [27]. As to the data by sex, the findings showed that $23 \%$ of the men and $52.1 \%$ of the women were of normal weight, $4.8 \%$ of the men and $9 \%$ of the women were overweight, $3 \%$ of the men and $7.2 \%$ of the women were underweight, and $0.6 \%$ of the women were obese (Figure 1). 


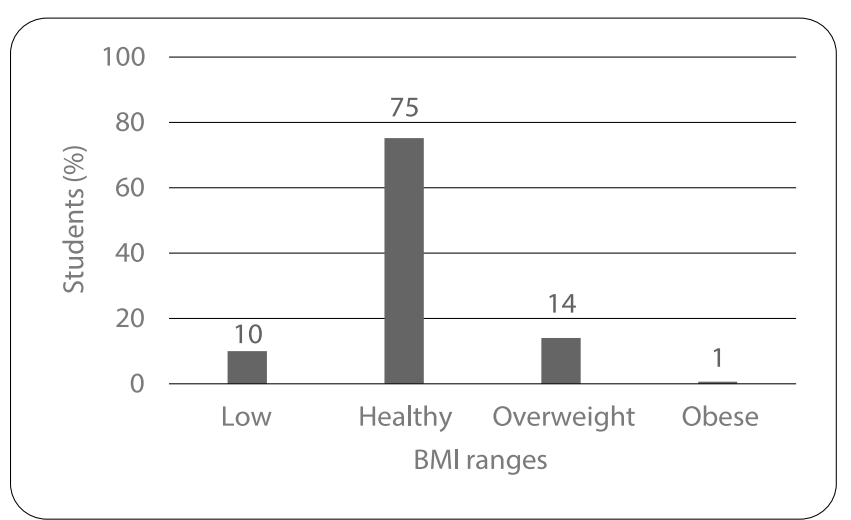

Figure 1. Body Mass Index (BMI)

As for the blood chemistry, $98 \%$ of students had fasting blood glucose levels within the normal parameters according to the recommendations of the American Diabetes Association (ADA) [2] and $2 \%$ of the population showed levels of pre-diabetes (Figure 2). Of the 165 students, an equivalent 30\% suffered hypercholesterolemia, $18 \%$ of students had hypertriglyceridemia (Figure 3), 17\% showed low HDL levels (Figure 4), and 67\% LDL level (Figure 5), based on the parameters of the American Heart Association (AHA) [1]. These results, in a population within an age range of 16 to 21, may be related to genetic factors or even be linked to poor nutritional habits. Of all the cases studied, 75 students $(40 \%)$ could in the future present a high risk to develop Metabolic Syndrome. Furthermore, $60 \%$ of the population studie said they did not engage in any physical activity, especially women (44.70\%). It should be noted that the remaining $40 \%$ perform a physical activity below the standardized levels of the WHO for the prevention of non-communicable diseases [28].

\section{DISCUSSION}

These results show us that diabetes mellitus and the metabolic syndrome are not diseases of older adults, due to the fact that cases were found in the study population aged 16-21 years, including individuals with high cardiovascular risk factors such as hypertriglyceridemia, hypercholesterolemia, obesity even $2 \%$ of these cases had pre-diabetes. These results are really important because, if they are not corrected in time, the damage caused can be irreversible.

These findings suggest that it is important to reinforce a culture of healthy habits in our population, characterized by a healthy diet and greater physical activity, which were two of the main alternatives for the prevention of non-communicable diseases such as obesity, diabetes and the metabolic syndrome [18, 26].

The metabolic syndrome, seen in its general context, is made up of a group of cardio-metabolic risk factors; and the identification of one or more elements thereof,

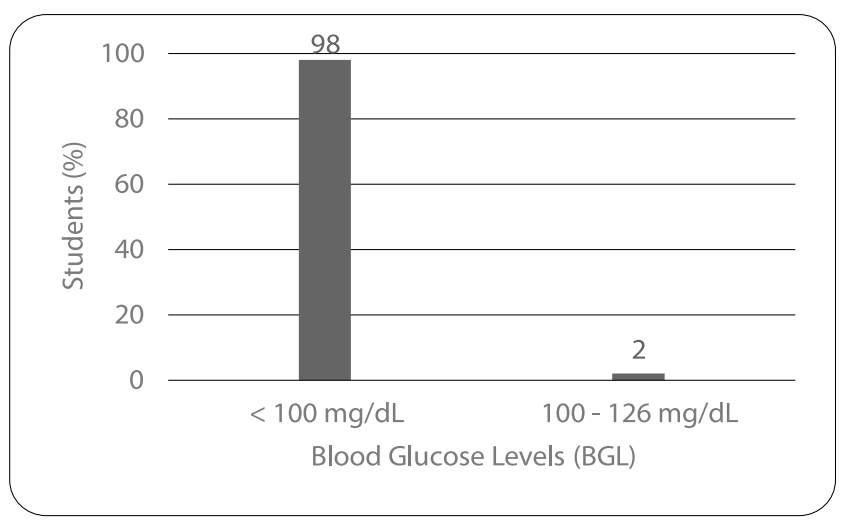

Figure 2. Blood Glucose Levels (BGL)

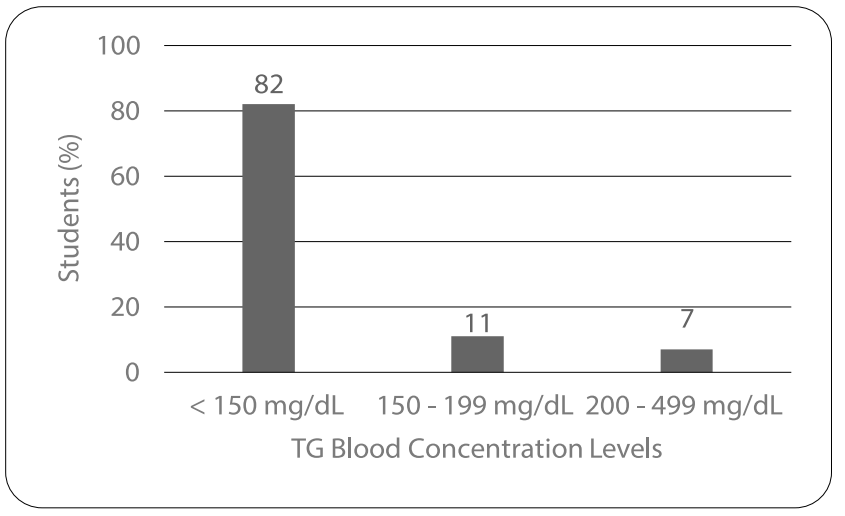

Figure 3. Triglicerides (TG) concentration levels in blood

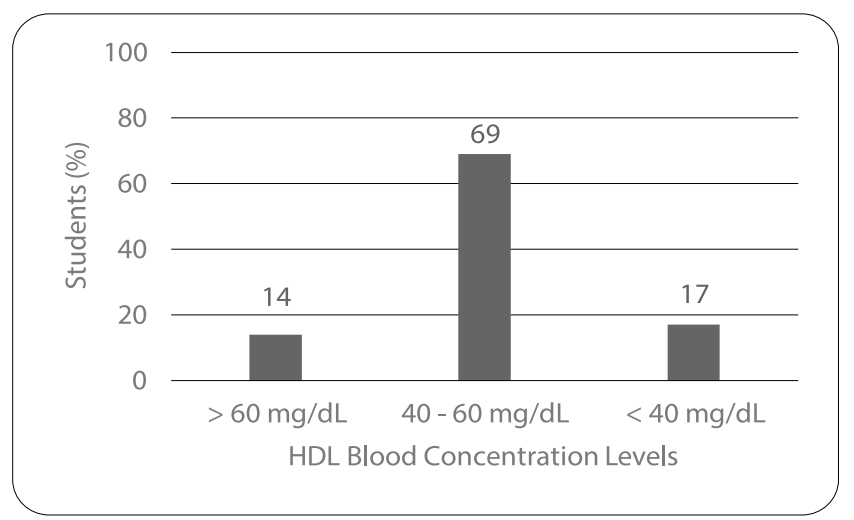

Figure 4. High-Density Lipoprotein (HDL)/ blood concentration levels

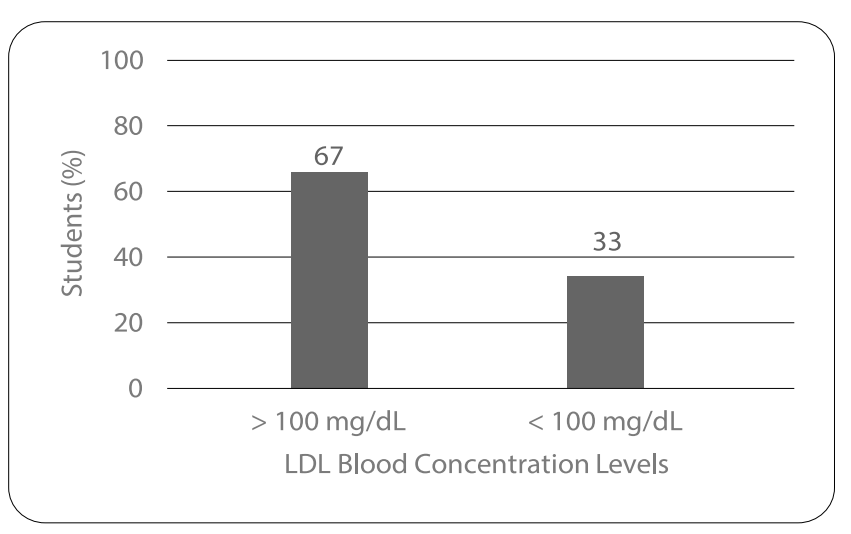

Figure 5. Low-Density Lipoprotein (LDL)/ blood concentration levels 
justifies the monitoring and clinical management of the patient since this risk could increase. This is why it is important to conduct a cardio-metabolic follow-up and control at an early age, since it would be possible to avoid the diseases with the highest mortality worldwide, which are due to these risks [19].

According to the National Nutritional Situation Survey (Encuesta Nacional de Situación Nutricional - ENSIN) [16] a nutritional survey was carried out to acquire representative information on the most relevant aspects of the food and nutrition situation, based on geographical (departmental) zones throughout Colombia, and to suggest the possible determinants that would explain the problems observed. Anthropometric measures were used for this study, in ages between 0 and 64 years, covering a population of 52,000 households, in 5,000 segments of urban and rural areas of 313 municipalities, with some of the following indicators: nutritional status by anthropometric values, household food security, dietary intake, eating habits, physical activity, sedentary life patterns, self-perception of body weight, time spent watching TV, among others [16].

Similar to the study carried out by the ENSIN 2015, which shows that only half of the Colombian population performs any physical activity, as recommended by the WHO [26]. Evidence can be observed of a prevalence of overweight among adults of $37.7 \%$ and an obesity index present in one in five members of this group, more common in women than in men. When adding these values, we have that a total of $56.4 \%$ of the Colombian population is overweight or obese, by reason of an inadequate food intake and sedentary lifestyle, which represents a 5.2 points increase compared to the ENSIN 2010. These findings match the information obtained in the recently presented paper, on how unhealthy eating habits, based on high consumption of carbohydrates, sausages and cold meats and low consumption of vegetables and fruits, as well as sedentary lifestyle, are conducive to the development of obesity, especially in women vis-à-vis men [16].

From the scope of public health, obesity has been considered a serious problem since one of the main complications of this disease are the insulin resistance and type II diabetes. It is estimated that for each increase of $7 \mathrm{~kg}$ of weight, the risk of diabetes increases by 50\% [3]. According to the Panamerican Health Organization (PAHO), if no action is taken, it is expected that by the year 2040 the number of Diabetes Mellitus patients will increase to about $110,000,000$ inhabitants in the Americas, as opposed to $62,000,000$ in 2016. The latest report shows that people with diabetes can lead a long and healthy life if detected early and well managed. However, in some countries in the Americas, up to $40 \%$ of individuals with diabetes are not aware of it, and 50 to $70 \%$ do not have an adequate control of their blood glucose levels, which is why the education and promotion of healthy habits, from an early age, is really important [2, 6, 29].

Amidst the studied sample, $13.93 \%$ of young people were overweight and the $0.6 \%$ was within obesity ranges (risk factors for developing a metabolic syndrome) and they both, were classified according to the criteria suggested by Grundy et al. [6]. Young adults with these conditions taking part in the institution's medical program, together with the support of the University Welfare Department, were informed about their situation based on the results obtained, in order to take measures for an appropriate health management behavior, suggesting their attendance to a medical consultation with the relevant specialist. Furthermore, through a meeting with the University's steering committee and the support of the Students Fund, it was possible to comprehensively address the management of young adults with risk factors for chronic non-communicable diseases.

Physical activity and sports are beneficial for the organic and psychological health of individuals in different settings, to protect the body from cardiovascular, respiratory and musculoskeletal factors. However, modern society's lifestyle is characterized by inactivity and a sedentary lifestyle. With emphasis placed upon the population of medical and higher education students, studies have been carried out ascertaining the high sedentary rate of this group of people, and establishing the main cause to be the lack of time and the fatigue of long hours devoted to both the university and to rotating internship at hospitals [5, 17]

In order to become an institution with adequate habits and healthy lifestyles, we investigated the different food outlets at the La Sabana University, and which was the main food consumed by young people. We evidenced a low consumption of fruits and vegetables and a high intake of other foods high in carbohydrates, such as pizzas, hot dogs, hamburgers, carbonated soft drinks, snacks, among others. In addition, we have studied the consumption of food of the medical students in their different hospital-rotation areas and, in addition, we found evidence of an inadequate food intake characterized by imbalanced diets based on carbohydrates consumed at all times during the day.

This intervention confirms that the majority of the population studied eats unhealthy foods. And it is for this reason that the university decided to reinforce the culture of healthy habits, by offering more menu alternatives that include the consumption of fruits and vegetables, as well as a restructuring of the food and beverage points, to promote an adequate nutrition, in accordance with the provisions set forth in article 4 
of Law 1355 of October 14, 2009 [4]. In addition, and taking into account the findings obtained on the previously described health problems in the students, the university decided to establish a Physical Fitness Center (CAF) - a space created to promote healthy lifestyles through physical activity, by developing and strengthening values such as discipline, teamwork, solidarity, self-esteem, work well done, among others. With this, the objective is to make a healthy use of free time and facilitate the integration of our University Community [24].

\section{CONCLUSIONS}

Malnutrition is associated with a wide range of serious health complications and an increased risk of prematurely contracting diseases, including diabetes and heart disease. These complications do not belong to a specific age group, such as older adults, since, as shown in the results of the study, increasing number of adolescents and young adults are suffering from overweight, obesity and its complications, and such results are observed, mainly, in medical students. It is therefore important to carry out an adequate followup and early cardio-metabolic monitoring based on a healthy diet and adequate physical activity With this, early establishment of various chronic noncommunicable diseases could be avoided.

Finally, highlight the fact that the prevention of these entities are not only an individual responsibility; therefore, La Sabana University encourages other institutions at school and higher education levels in order to apply these preventive measures to „make the healthy option the easiest option to take".

\section{Funding}

This work was financed by the authors and with funds alocated by the Research Department for the project.

\section{Acknowledgements}

This project (EICEA-68-2013) was done with the support of the Research Department of La Sabana University in Bogota, Colombia.

\section{Conflicts of interest}

None declared.

\section{REFERENCES}

1. ACC/AHA. Guideline on the Management of Blood Cholesterol. Am Coll Cardiol Hear Assoc. 2018; https:// doi.org/10.1161/CIR.0000000000000625

2. American Diabetes Association. Summary of revisions to the 2014 Clinical Practice Recommendations. Diabetes Care. 2014;37. https://doi.org/10.2337/ dc14-S004
3. Bray GA, Frühbeck $G$, Ryan DH, Wilding JPH.: Management of obesity. The Lancet. 2016. https://doi. org/10.1016/S0140-6736(16)00271-3

4. Congreso de la República de Colombia. LAW 1355, 2009. Diario Oficial 2009.

5. Contreras JJ, Espinoza RM, Dighero B, Drullinsky D, Liendo R, Soza-Rex F.: Sedentary attitude and related factors in medical school students. Rev Andaluza Med del Deport. 24 de junio de 2009;43(2S):176. https://doi. org/10.36104/amc.2018.1400

6. Grundy SM, Cleeman JI, Daniels SR, Donato $K A$, Eckel RH, Franklin BA, et al.: Diagnosis and Management of the Metabolic Syndrome. Circulation. 2005;112(17):2735-2752. CIRCULATIONAHA.105.169404

7. Gutiérrez M, Beneit S.: Application of new technologies to the analysis of the body composition. Vol. 1. Nutricion Hospitalaria. 2011;1:120-125. https:/doi. org $/ 10.21149 / 7891$

8. Habib SH, Saha S.: Burden of non-communicable disease: Global overview. Diabetes Metab Syndr Clin Res Rev. 2010;4(1):41-47. https://doi.org/10.1016/j. dsx.2008.04.005

9. Henningsen M.: Dietary habits in adolescence related to sociodemographic factors, physical activity and selfesteem. Thesis. Department of Social Work and Health Science, Faculty of Social Science and Technology Management, Norwegian University of Science and Technolog. 2011. http://hdl.handle.net/11250/267803

10. Low LK.: Guidelines for adolescent preventive services (GAPS). J Midwifery Women's Health. 2010:231-233,https://doi.org/10.1016/S15269523(03)00071-0

11. Maddaleno M, Morello P, Infante-Espinola F.: Health and development of adolescents and young adults in Latin America and the Caribbean: Challenges for the next decade. Salud Publica Mex. 2003;45,Suppl, S132-9, https://pubmed.ncbi.nlm.nih.gov/12602156/

12. Melnyk BM, Jacobson D, Kelly S, Belyea M, Shaibi $G$, Small $L$, et al.: Promoting healthy lifestyles in high school adolescents: A randomized controlled trial. Am J Prev Med. 2013; https://doi.org/10.1016/j. amepre.2013.05.013

13. Méndez Castellano H, Bosch V LBMF.: Clinical protocoles for integral atention of adolescents. IMAN services strategies. Enfermedades no Transm. 2012;1:116.

14. Merino BM, Briones EG, Aznar-Lain S, Webster T: Physical activity and health in children and adolescents. A guide for all adults involved in educating young people. Ministerio Español de Sanidad y Consumo, 2008 p. 101.

15. National Administration Department of Statistics (DANE). Population projections 2005 -2020. Colombia. National and regional mortality charts from 1985 to 2020. Proyecciones de Población 2005-2020. 2007;4-22.

16. National Ministry of Health SP and SP. National Report about Nutritional Situation ENSIN. Encuesta Nacional de la Situación Nutricional ENSIN- 2015. 2017. 
17. Navarrete Mejia PJ, Parodi García JF, Vega García E, Pareja Cruz A, Benites Azabache JC.: Sedentarism factors in young university students. Perú, 2017. Horiz Médico. 15 de marzo de 2019;19(1):46-52.

18. Nianogo RA, Arah OA.: Impact of Public Health Interventions on Obesity and Type 2 Diabetes Prevention: A Simulation Study. Am J Prev Med. 2018; https://doi.org/10.1016/j.amepre.2018.07.014

19. Nolan PB, Carrick-Ranson G, Stinear JW, Reading $S A$, Dalleck LC.: Prevalence of metabolic syndrome and metabolic syndrome components in young adults: A pooled analysis. Prev Med Reports. 2017;7:211-215, https://doi.org/10.1016/j.pmedr.2017.07.004

20.Obregón M.C., Celis L.G., Ayala J.J., Domínguez M.T., Giratá M.V., Almonacid C.C.: Factores promotores de cultura nutricional en adolescentes y adultos jóvenes de la Universidad de La Sabana. Salud Uninorte 2013;29(3):374-383.

21. Panamerican Health Organization PAHO. Adolescent and Youth Regional Strategy and Plan of Action. Vol. 10. 2010.

22.Pandita A, Sharma D, Pandita D, Pawar S, Tariq M, Kaul A.: Childhood obesity: Prevention is better than cure. Diabetes, Metabolic Syndrome and Obesity: Targets and Therapy. 2016. https://doi.org/10.2147/ DMSO.S90783

23. Rodrigues PRM, Luiz RR, Monteiro LS, Ferreira MG, Gonçalves-Silva RMV, Pereira RA.: Adolescents' unhealthy eating habits are associated with meal skipping. Nutrition. 2017;42:114-120.el https:/doi. org/10.1016/j.nut.2017.03.011

24. Sabana U de La. Physical Fitness Center, University de La Sabana [Internet]. 2017 [cited February 8 $8^{\text {th }} 2020$ ]. Available at: https://www.unisabana.edu.co/bienestaruniversitario/deportes/centro-de-acondicionamientofisico

25. U.S. Preventive Services Task Force. The Guide to Clinical Preventive Services 2014. Agency for Healthcare Research and Quality. 2014.

26. Wiklund P.: The role of physical activity and exercise in obesity and weight management: Time for critical appraisal. Journal of Sport and Health Science. 2016;5(2):151-154. https://doi.org/10.1016/j. jshs.2016.04.001

27. World Health Organization WHO. Obesity and Overweight. 2020.

28. World Health Organization WHO. Physical activity in adults. [February $\left.8^{\text {th }} 2020\right]$. p. Para los adultos de este grupo de edades, la activ. Disponible en: https://www. who.int/dietphysicalactivity/factsheet_adults/en/

29. World Health Organization WHO. The number of people with diabetes in the Americas has tripled since 1980. 2016. [cited february $8^{\text {th }} 2020$ ]. Available at: https://www.paho.org/en/news/6-4-2016-numberpeople-diabetes-americas-has-tripled-1980

Received: 16.09 .2020

Accepted: 05.03.2021 